\title{
An Experimental Comparison of Several PWM Controllers for a Single-Phase AC-DC Converter
}

\author{
Dimitrios Karagiannis, Eduardo Mendes, Alessandro Astolfi, and Romeo Ortega
}

\begin{abstract}
An experimental comparative study of various controllers for a class of ac-dc converters, known as power factor precompensators (PFP) is presented. The control objective is to robustly regulate the output voltage to a desired constant level in the presence of variations in the load, while retaining a unit power factor at the input, i.e., the input current should follow in frequency and in phase the input voltage. Several control schemes are presented and compared via experiments.
\end{abstract}

Index Terms-AC-DC power conversion, adaptive control, nonlinear systems, power factor correction, pulsewidth modulation.

\section{INTRODUCTION}

$\mathbf{P}$ OWER FACTOR PRECOMPENSATORS (PFP) are an important class of switched ac-dc converters. As their name suggests, their main function is to achieve a nearly unit power factor by drawing a sinusoidal current that is in phase with the source voltage, thus eliminating the reactive power and the harmonic interference with other equipment operating off the same source.

More precisely, the control objective is twofold. First, the input current should track a sinusoidal reference signal that is in phase with the input voltage. Second, the output voltage should be driven to a desired constant level. An additional requirement is robustness against variation of the system parameters and in particular of the load, which is usually unknown.

Since the amplitude of the input current determines explicitly the dc output voltage, one may satisfy both objectives in a single current control loop, which typically comprises a hysteresis or sliding-mode controller (see [1, Ch. 18] for a general description and [2] for a design example).

The main drawback of these controllers is that they require very high switching frequency (typically few hundred kilohertz) leading to high converter losses. In this brief, we are interested in pulsewidth modulation (PWM) control techniques, which are based on averaged models [3] and can be implemented using lower switching frequency (e.g., $10 \mathrm{kHz}$ ). Furthermore, the issue of robustness against variations of the load is treated by appropriate adaptive schemes. This allows to avoid the

Manuscript received April 12, 2002. Manuscript received in final form February 26, 2003. Recommended by Associate Editor J. Chiasson. This work was supported in part by the European TMR network NACO2.

D. Karagiannis is with the Department of Electrical and Electronic Engineering, Imperial College, London SW7 2BT, U.K. (e-mail: d.karagiannis@imperial.ac.uk).

A. Astolfi is with the Department of Electrical and Electronic Engineering, Imperial College, London SW7 2BT, UK.

E. Mendes is with the Institut National Polytechnique de Grenoble, ESISAR, 26902 Valence, France (e-mail: Eduardo.Mendes@esisar.inpg.fr).

R. Ortega is with the Laboratoire des Signaux et Systèmes, SUPELEC, 91192

Gif-sur-Yvette, France (e-mail: Romeo.Ortega@1ss.supelec.fr).

Digital Object Identifier 10.1109/TCST.2003.815549

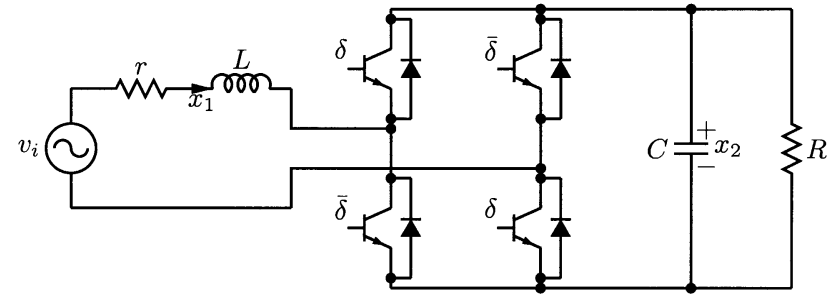

Fig. 1. Diagram of the full-bridge boost PFP circuit.

use of an output current sensor, thus making the practical implementation more attractive.

The paper is organized as follows. In Section II, we analyze the model of the PFP, define the desired equilibrium regime and specify the control strategy. In Section III, we propose various control schemes that achieve the control objectives. An adaptive version for each controller is considered separately. In Section IV, we present the experimental results and compare the performance of the proposed controllers. Conclusions are given in Section V.

\section{THE PFP CIRCUIT}

We consider the full-bridge boost PFP circuit shown in Fig. 1, which consists of two pairs of transistor-diode switches working in a complementary way. The switching signal $\delta$ is generated by a PWM circuit and takes values in the finite set $\{-1,1\}$. The averaged model of the PFP can be obtained using Kirchhoff's laws and is given by the equations

$$
\begin{aligned}
& L \dot{x}_{1}=-u x_{2}-r x_{1}+v_{i}(t) \\
& C \dot{x}_{2}=u x_{1}-\frac{1}{R} x_{2}
\end{aligned}
$$

where $v_{i}(t)=E \sin (\omega t)$ is the source voltage, $x_{1}$ is the input (inductor) current, $x_{2}>0$ is the output (capacitor) voltage, $L, C, r$, and $R$ are positive constants, representing the inductance, capacitance, parasitic resistance, and load resistance, respectively, and $u \in[-1,1]$ is the duty ratio of the PWM. ${ }^{1}$

The desired input current in steady state is

$$
x_{1}^{\star}(t)=I_{d} \sin (\omega t)
$$

for some $I_{d}>0$ yet to be specified. Substituting (3) into (1) yields the steady-state value for $u x_{2}$, namely

$$
u^{\star}(t) x_{2}^{\star}(t)=v_{i}(t)-r x_{1}^{\star}(t)-L \dot{x}_{1}^{\star}(t) .
$$

\footnotetext{
${ }^{1}$ Note that the exact model of the PFP is described by the same equations, if
} we replace $u$ with $\delta$. 
Substituting (3) and (4) into (2) written for $x_{2}=x_{2}^{\star}$ yields

$$
\begin{aligned}
C \dot{x}_{2}^{\star} x_{2}^{\star} & =-\frac{1}{R} x_{2}^{\star 2}+\frac{\left(E-r I_{d}\right) I_{d}}{2} \\
& -\frac{\left(E-r I_{d}\right) I_{d}}{2} \cos (2 \omega t)-\frac{L I_{d}^{2} \omega}{2} \sin (2 \omega t) .
\end{aligned}
$$

The steady-state solution of (5) can be directly computed and is given by

$$
x_{2}^{\star 2}(t)=\frac{\left(E-r I_{d}\right) R I_{d}}{2}+A \sin (2 \omega t+\phi),
$$

where

$$
\begin{aligned}
& A=\frac{R I_{d}}{2} \sqrt{\frac{\left(L I_{d} \omega\right)^{2}+\left(E-r I_{d}\right)^{2}}{1+(R C \omega)^{2}}}, \\
& \phi=\arctan \frac{E-r I_{d}-R C \omega L I_{d} \omega}{L I_{d} \omega+R C \omega\left(E-r I_{d}\right)}
\end{aligned}
$$

Hence, in steady state, $x_{2}^{\star 2}(t)$ consists only of a dc term and a second-order harmonic. The rms of $x_{2}^{\star}(t)$, denoted by $V_{d}$, is given by

$$
V_{d}=\sqrt{\frac{\left(E-r I_{d}\right) R I_{d}}{2}} .
$$

From (7), solving for $I_{d}$ yields the solutions

$$
I_{d}=\frac{E}{2 r} \pm \sqrt{\frac{E^{2}}{4 r^{2}}-\frac{2 V_{d}^{2}}{r R}},
$$

which are real if and only if

$$
\frac{V_{d}}{E} \leq \sqrt{\frac{R}{8 r}}
$$

Selecting the smallest solution, which corresponds to minimum power (i.e., minimum converter losses), the amplitude of the input current that drives the output voltage to the desired level $V_{d}$ is given by

$$
I_{d}=\frac{E}{2 r}-\sqrt{\frac{E^{2}}{4 r^{2}}-\frac{2 V_{d}^{2}}{r R}} .
$$

Note that the same solution has been obtained by a similar analysis in [4].

We conclude that by controlling the input current so that it tracks the signal (3), where $I_{d}$ is given by (8), we can achieve both control objectives, namely unit power factor and output voltage regulation, provided that the closed-loop system is asymptotically stable.

Remark 1: The main drawback of this approach, as (7) and (8) reveal, is the sensitivity of the output voltage to the parameters $r$ and $R$. The dependence on $R$ poses a significant problem, since in many applications the load is unknown or time-varying. This obstacle can be overcome by adding an adaptation scheme either on $I_{d}$ or on $R$.

Remark 2: In practice, the second-order harmonic in (6) can be neglected, since its amplitude is much smaller than the dc term, and so the average of $x_{2}^{\star}(t)$ can be approximated by the rms value. For instance, for the set of parameters used in the experiments (see Table I in Section IV), and for $V_{d}=200 \mathrm{~V}$, we have $A=1330 \ll V_{d}^{2}=40000$ and $\operatorname{avg}\left(x_{2}^{\star}(t)\right)=199.986 \approx$ $V_{d}=200$.

Remark 3: Recall that the control signal $u$ must satisfy the constraint $|u| \leq 1$. A necessary condition for the existence of this control is derived in [4] and shows that the PFP can only work as a boost converter.

\section{CONTROL LAWS}

In this section, we propose four controllers for the regulation of the input current. First, we consider the case where all the parameters are known, so we can use (8) to compute the reference current. An adaptive scheme will later be added to deal with the uncertainty in the load.

\section{A. The Known Parameter Case}

The control designs that will be compared are the following.

1) Passivity-Based (PB): In [4], the passivity-based controller described by

$$
u=\frac{1}{x_{2 d}}\left[v_{i}-r x_{1}-L \dot{x}_{1}^{\star}-K_{1}\left(x_{1}^{\star}-x_{1}\right)\right]
$$

where the auxiliary variable $x_{2 d}>0$ is the solution of

$$
C \dot{x}_{2 d}=u x_{1}^{\star}-\frac{1}{R} x_{2 d}-K_{2}\left(x_{2 d}-x_{2}\right)
$$

with $x_{1}^{\star}$ given by (3), has been proposed. This controller renders the closed-loop system exponentially stable with the energy function

$$
V=\frac{L}{2}\left(x_{1}^{\star}-x_{1}\right)^{2}+\frac{C}{2}\left(x_{2 d}-x_{2}\right)^{2}
$$

whose time derivative satisfies

$$
\begin{aligned}
\dot{V} & =-K_{1}\left(x_{1}^{\star}-x_{1}\right)^{2}-\left(R^{-1}+K_{2}\right)\left(x_{2 d}-x_{2}\right)^{2} \\
& \leq-2 \frac{\min \left(K_{1}, R^{-1}+K_{2}\right)}{\max (L, C)} V .
\end{aligned}
$$

Hence, for $K_{1}>0$ and $K_{2}>0, x_{1}$ and $x_{2}$ converge exponentially to $x_{1}^{\star}$ and $x_{2 d}$, respectively. Moreover, $x_{2 d}$ converges in average to $V_{d}$ provided that (8) holds (see [4], where this has been proved).

2) Feed-Forward $(F F)$ : If we replace in the control law (9) the auxiliary state $x_{2 d}$ with the output voltage $x_{2}$, we obtain the simplified controller

$$
u=\frac{1}{x_{2}}\left[v_{i}-r x_{1}^{\star}-L \dot{x}_{1}^{\star}-K_{1}\left(x_{1}^{\star}-x_{1}\right)\right] .
$$

Substituting (11) into (1) and defining

$$
\tilde{x}_{1}=x_{1}^{\star}-x_{1}
$$

yields the linearized error dynamics

$$
L \dot{\tilde{x}}_{1}=-\left(r+K_{1}\right) \tilde{x}_{1}
$$

which is exponentially stable for any $K_{1}>-r$. Hence, the time response of $x_{1}$ is given by

$$
x_{1}(t)=x_{1}(0) \exp \left(-\frac{r+K_{1}}{L} t\right)+I_{d} \sin (\omega t) .
$$


Assuming that $x_{1}$ takes its steady-state value $x_{1}(t)=$ $I_{d} \sin (\omega t)$ immediately and substituting into (2) yields

$$
\begin{aligned}
\dot{x}_{2}= & -\frac{1}{R C} x_{2}+\frac{\left(E-r I_{d}\right) I_{d}}{2 C x_{2}} \\
& -\frac{\left(E-r I_{d}\right) I_{d}}{2 C x_{2}} \cos (2 \omega t)-\frac{L I_{d}^{2} \omega}{2 C x_{2}} \sin (2 \omega t) .
\end{aligned}
$$

Rewriting (13) in the variable $x_{2}^{2}$ yields the linear dynamics

$$
\begin{aligned}
\dot{x}_{2}^{2}= & -\frac{2}{R C} x_{2}^{2}+\frac{\left(E-r I_{d}\right) I_{d}}{C} \\
& -\frac{\left(E-r I_{d}\right) I_{d}}{C} \cos (2 \omega t)-\frac{L I_{d}^{2} \omega}{C} \sin (2 \omega t) .
\end{aligned}
$$

The steady-state solution of (14) is given by (6), implying that $x_{2}$ converges (in average) to the desired voltage (7).

Remark 4: The FF controller is actually a feedback linearising controller. The term "feed-forward" arises from the use of $x_{1}^{\star}$ and $\dot{x}_{1}^{\star}$ in the control law (11).

3) Feedback Linearization (FL): A static feedback control law that linearises the input-output behavior of the system with output the inductor current is described by

$$
u=\frac{1}{x_{2}}\left(v_{i}-r x_{1}-w\right) .
$$

It is easy to see that the resulting linearized system with input $w$ and output $x_{1}$ is an integrator. Thus, if we apply proportional control, i.e.

$$
w=K_{1}\left(x_{1}^{\star}-x_{1}\right)
$$

with $K_{1}>0$, then it can be shown that the closed-loop system is asymptotically stable. The closed-loop dynamics of $x_{1}$ are given by

$$
L \dot{x}_{1}=-K_{1} x_{1}+K_{1} I_{d} \sin (\omega t) .
$$

Hence, in steady state

$$
x_{1}(t)=\frac{1}{\sqrt{1+\mu^{2}}} I_{d} \sin (\omega t-\arctan \mu)
$$

where $\mu=L \omega / K_{1}$. Note that, in this case, there is a steady-state error, ${ }^{2}$ which decreases as we increase the gain $K_{1}$. As a result, the error $x_{1}^{\star}-x_{1}$ (and consequently the output voltage error) can be made arbitrarily small by selecting $K_{1}$ sufficiently large.

4) Internal Model (IM): If instead of $x_{1}$ we take as output the signal

$$
z=u x_{2}
$$

which corresponds to the input voltage of the transistor-diode bridge, then the system can be linearized by means of the dynamic controller ${ }^{3}$

$$
\dot{u}=\frac{1}{x_{2}}\left(-\frac{u^{2} x_{1}}{C}+w\right) .
$$

${ }^{2}$ In particular, there is a negative phase-shift, i.e., the input current leads the input voltage.

${ }^{3}$ To prove this fact, consider the dynamics of $z: \dot{z}=\dot{u} x_{2}+\left(\left(u^{2} x_{1}\right) / C\right)-$ $(1 / R C) z$.
The linearized system is described by

$$
\begin{aligned}
{\left[\begin{array}{c}
\dot{x}_{1} \\
\dot{z}
\end{array}\right] } & =\left[\begin{array}{cc}
-\frac{r}{L} & -\frac{1}{L} \\
0 & -\frac{1}{R C}
\end{array}\right]\left[\begin{array}{c}
x_{1} \\
z
\end{array}\right]+\left[\begin{array}{c}
\frac{v_{i}}{L} \\
w
\end{array}\right] \\
y & =\left[\begin{array}{ll}
0 & 1
\end{array}\right]\left[\begin{array}{c}
x_{1} \\
z
\end{array}\right] .
\end{aligned}
$$

Hence, the transfer function from $w$ to $y$ is given by ${ }^{4}$

$$
G(s)=\frac{1}{s+\frac{1}{R C}} .
$$

It is clear from the analysis in Section II that we can achieve the control objectives by forcing $y(t)$ to track the signal

$$
y^{\star}(t)=z^{\star}(t)=v_{i}-r x_{1}^{\star}-L \dot{x}_{1}^{\star} .
$$

To this end, consider the error

$$
e=y^{\star}-y=v_{i}-r x_{1}^{\star}-L \dot{x}_{1}^{\star}-u x_{2}
$$

and the controller

$$
K(s)=\frac{w(s)}{e(s)}=k \frac{s^{2}+a s+b}{s^{2}+\omega^{2}}
$$

where $k>0, a>0$, and $b>0$ are design parameters. Then, for sufficiently large $k$, the closed-loop system is asymptotically stable. Moreover, by the presence of the poles at $s= \pm j \omega$, we conclude that $y$ converges to $y^{\star}$ and hence $x_{1}$ converges to $x_{1}^{\star}$.

Remark 5: The choice of the controller (24) is motivated by the internal model principle and the fact that the reference signal $y^{\star}(t)$ is a sinusoid of frequency $\omega$. Furthermore, the zeros have been inserted to ensure stability.

Remark 6: In practice, in order to compensate for unmodeled dynamics, it is necessary to add to the reference signal (22) a correction term proportional to the error $x_{1}^{\star}-x_{1}$, i.e.

$$
z^{\star}(t)=v_{i}-r x_{1}^{\star}-L \dot{x}_{1}^{\star}-K_{1}\left(x_{1}^{\star}-x_{1}\right) .
$$

To incorporate this into our linear formulation, consider instead of (20) the output

$$
y=\left[\begin{array}{ll}
-K_{1} & 1
\end{array}\right]\left[\begin{array}{c}
x_{1} \\
z
\end{array}\right]
$$

and the reference

$$
y^{\star}(t)=v_{i}-r x_{1}^{\star}-L \dot{x}_{1}^{\star}-K_{1} x_{1}^{\star} .
$$

The transfer function from $w$ to $y$ is now given by

$$
G(s)=\frac{s+\frac{\left(r+K_{1}\right)}{L}}{\left(s+\frac{r}{L}\right)\left(s+\frac{1}{R C}\right)} .
$$

It can be straightforwardly verified (e.g., using root locus) that, for sufficiently large $k>0$, the closed-loop system is asymptotically stable provided $K_{1}>-r$.

\section{B. The Unknown Parameter Case}

So far, we have assumed that the parameters $r$ and $R$ were known and so we could control the output voltage indirectly via (8). In practice, however, the load $R$ is usually unknown or timevarying. To overcome this robustness problem, it is necessary to add an adaptation scheme either to the parameter $R$ or to the parameter $I_{d}$.

\footnotetext{
${ }^{4}$ Note the cancellation of the stable pole at $s=-r / L$.
} 
We describe three adaptation schemes, for which (under some mild assumptions) asymptotic stability can be proved. The first one is an estimator proposed in [4] for the PB controller. The other two are based on the recently developed adaptive control methods of nonlinear proportional integral (NLPI) control and immersion and invariance (I\&I) (see [5], [6] for further details).

1) PB Estimator: For the PB controller (9), (10), a projection estimator for $\theta=1 / R$ has been proposed in [4], namely

$$
\dot{\hat{\theta}}= \begin{cases}\gamma x_{2 d}\left(x_{2 d}-x_{2}\right), & \hat{\theta} \geq \epsilon>0 \\ 0, & \hat{\theta}<\epsilon\end{cases}
$$

(where $\hat{\theta}$ denotes the estimate of $\theta$ ) and has been shown to render the equilibrium $\left(x_{1}, x_{2}\right)=\left(x_{1}^{\star}, x_{2 d}\right)$ asymptotically stable, assuming that $r=0$.

2) Nonlinear PI (NLPI) Control: Invoking time-separation arguments, we can assume that the dynamics of the output voltage are slow compared to the (closed-loop) dynamics of the input current ${ }^{5}$ and hence $x_{1}=x_{1}^{\star}$ at all times. ${ }^{6}$ Furthermore, we assume $r=0$. Then from (13), ignoring second-order harmonics, the dynamics of $x_{2}$ are given by

$$
\dot{x}_{2}=-\frac{1}{R C} x_{2}+\frac{E}{2 C x_{2}} I_{d} .
$$

Note that the assumption $r=0$ ensures the above system is affine in the "control" $I_{d}$.

The equilibrium $x_{2}=x_{2}^{\star}$ is rendered asymptotically stable by the dynamic control law

$$
\dot{I}_{d}=\alpha \frac{E}{2 x_{2}} \tilde{x}_{2}+\beta \dot{\tilde{x}}_{2}
$$

where $\alpha$ and $\beta$ are positive constants. To prove this fact, consider the positive definite function

$$
V=\frac{1}{2} C \tilde{x}_{2}^{2}+\frac{1}{2 \alpha}\left(\tilde{I}_{d}+\beta \tilde{x}_{2}\right)^{2}
$$

whose time derivative along the trajectories of (27) and (28) is given by

$$
\dot{V}=-\left(\frac{1}{R}+\frac{V_{d}}{R x_{2}}+\frac{\beta E}{2 x_{2}}\right) \tilde{x}_{2}^{2}
$$

which is negative semidefinite and hence the system (27), (28) is stable. Moreover, $\dot{V}=0$ implies that $\tilde{x}_{2}=0$ and so, by LaSalle's invariance principle [7], the system is asymptotically stable.

Remark 7: The difference between the controller (28) and a $\mathrm{PI}$ is that here the integral gain is not a constant, but a (nonlinear) function of $x_{2}$. In particular, it is proportional to the ratio $E / x_{2}$. Approximating this ratio by a constant results in the industrystandard PI controller. (14).

${ }^{5}$ We can validate this assumption by comparing the time constants of (12) and ${ }^{6}$ Note that, for this assumption to be valid, the dynamics of $I_{d}$ must be sufficiently slow.
Remark 8: The assumption $r=0$ in both designs can be replaced by the assumption

$$
r x_{1}=\epsilon \sin (\omega t)
$$

where $\epsilon$ is a positive constant. Then we can incorporate the voltage drop across $r$ into the source voltage by taking

$$
v_{i}(t)=(E-\epsilon) \sin (\omega t) .
$$

Note that this simple modification improves the experimental results significantly.

3) I\&I Control: Using the adaptive I\&I method [6] we can design a parameter estimator for both $R$ and $r$ provided that the system (1), (2) is bounded-input bounded-state (BIBS) stable. We now prove that the trajectories of (1), (2) are bounded for any $u$.

Consider the positive definite function

$$
V=\frac{1}{2} L x_{1}^{2}+\frac{1}{2} C x_{2}^{2}
$$

which corresponds to the electrical energy stored in the system. The time derivative of (29) along the trajectories of (1), (2) is given by

$$
\dot{V}=-r x_{1}^{2}+v_{i} x_{1}-\frac{1}{R} x_{2}^{2} .
$$

By adding the positive term $\left(x_{1} \sqrt{r / 2}-v_{i} / \sqrt{2 r}\right)^{2}$ to the right-hand side (RHS) we obtain

hence

$$
\dot{V} \leq-r x_{1}^{2}+\frac{r}{2} x_{1}^{2}+\frac{1}{2 r} v_{i}^{2}-\frac{1}{R} x_{2}^{2}
$$

$$
\dot{V} \leq-\frac{r}{2} x_{1}^{2}-\frac{1}{R} x_{2}^{2}+\frac{1}{2 r} v_{i}^{2}
$$

which shows that the trajectories remain bounded provided $r>$ 0 . Note also that the system (1), (2) is linearly parameterized with respect to the parameters

$$
\theta_{1}=r, \quad \theta_{2}=\frac{1}{R} .
$$

Following the adaptive I\&I methodology [6], we define the estimation errors

$$
\begin{aligned}
& z_{1}=\hat{\theta}_{1}+\beta_{1}\left(x_{1}\right)-\theta_{1}, \\
& z_{2}=\hat{\theta}_{2}+\beta_{2}\left(x_{2}\right)-\theta_{2}
\end{aligned}
$$

and the update laws

$$
\begin{aligned}
& \dot{\hat{\theta}}_{1}=\frac{1}{L} \frac{\partial \beta_{1}}{\partial x_{1}}\left[u x_{2}+x_{1}\left(\hat{\theta}_{1}+\beta_{1}\left(x_{1}\right)\right)-v_{i}\right] \\
& \dot{\hat{\theta}}_{2}=\frac{1}{C} \frac{\partial \beta_{2}}{\partial x_{2}}\left[-u x_{1}+x_{2}\left(\hat{\theta}_{2}+\beta_{2}\left(x_{2}\right)\right)\right] .
\end{aligned}
$$

The resulting error dynamics are given by

$$
\begin{aligned}
& \dot{z}_{1}=\frac{\partial \beta_{1}}{\partial x_{1}} \frac{x_{1}}{L} z_{1} \\
& \dot{z}_{2}=\frac{\partial \beta_{2}}{\partial x_{2}} \frac{x_{2}}{C} z_{2} .
\end{aligned}
$$


TABLE I

PARAMETER VALUES FOR THE PFP CiRCUIT

\begin{tabular}{c|c|c}
\hline Parameter & Value & Unit \\
\hline \hline$E$ & 150 & $\mathrm{~V}$ \\
\hline$\omega$ & $100 \pi$ & $\mathrm{rad} / \mathrm{sec}$ \\
\hline$r$ & 2.2 & $\Omega$ \\
\hline$L$ & 2.13 & $m \mathrm{H}$ \\
\hline$C$ & 1100 & $\mu \mathrm{F}$ \\
\hline$R$ & 87 & $\Omega$ \\
\hline
\end{tabular}

Hence, by choosing

$$
\begin{aligned}
& \beta_{1}\left(x_{1}\right)=-\kappa x_{1}^{2} \\
& \beta_{2}\left(x_{2}\right)=-\lambda x_{2}
\end{aligned}
$$

with $\kappa>0, \lambda>0$ constants and recalling that $x_{2}>0$ and $x_{1}$ is not identically zero, we obtain the asymptotically stable dynamics

$$
\begin{aligned}
& \dot{z}_{1}=-\frac{2 \kappa x_{1}^{2}}{L} z_{1} \\
& \dot{z}_{2}=-\frac{\lambda x_{2}}{C} z_{2} .
\end{aligned}
$$

Hence, $\hat{\theta}_{1}+\beta_{1}\left(x_{1}\right)$ converges to $\theta_{1}$ and $\hat{\theta}_{2}+\beta_{2}\left(x_{2}\right)$ converges to $\theta_{2}$.

Remark 9: The main advantage of the I\&I method is that it relies only on BIBS stability. In this case, we have seen that the trajectories of (1), (2) are bounded regardless of the input. Hence, the I\&I adaptation can be used in conjunction with any stabilising control law $u\left(x_{1}, x_{2}, r, 1 / R\right)$, which can thus be replaced by $u\left(x_{1}, x_{2}, \hat{\theta}_{1}+\beta_{1}, \hat{\theta}_{2}+\beta_{2}\right)$, where $\hat{\theta}_{1}, \hat{\theta}_{2}$ and $\beta_{1}, \beta_{2}$ are given by (30), (31) and (32), (33), respectively.

\section{EXPERIMENTAL RESULTS}

The control algorithms described in Section III have been implemented on an experimental system with the parameters shown in Table I. A diagram of the experimental setup is given in Fig. 2. The controller design parameters are: $K_{1}=15, K_{2}=1$, $k=4600, a=1200, b=2 \cdot 10^{5}$. The switching frequency of the PWM is $13 \mathrm{kHz}$. The measurements are filtered using low-pass filters with cut-off frequency $7 \mathrm{kHz}$.

The experiments aim to exhibit the behavior of the controllers with respect to step changes in the desired output voltage $V_{d}$ and also with respect to step changes in the load resistance $R$. In particular, the set-points for the output voltage are $V_{d}=160 \mathrm{~V}$ and $V_{d}=200 \mathrm{~V}$, while the load $R$ can be switched from 87 to $51 \Omega .^{7}$

\section{A. The Known Parameter Case}

To begin with, we consider the nonadaptive versions of the controllers, i.e., the parameters $r$ and $R$ are known and $I_{d}$ is computed from (8). Fig. 3 shows the filtered ${ }^{8}$ response to an

\footnotetext{
${ }^{7}$ Such converters with wide load and large output can be found, for instance, in medical-use X-ray generator applications.

${ }^{8}$ For the comparative graphs to be legible, the harmonics of the output voltage were removed by a moving average filter.
}

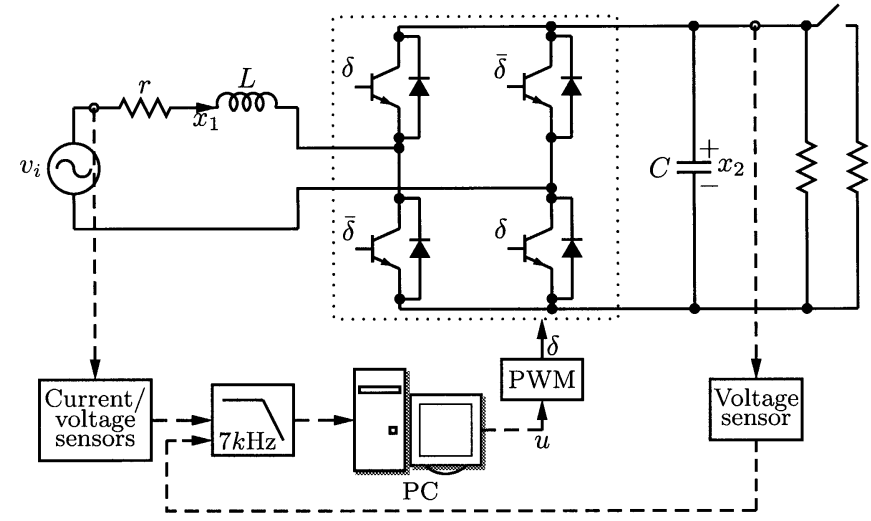

Fig. 2. Experimental setup.

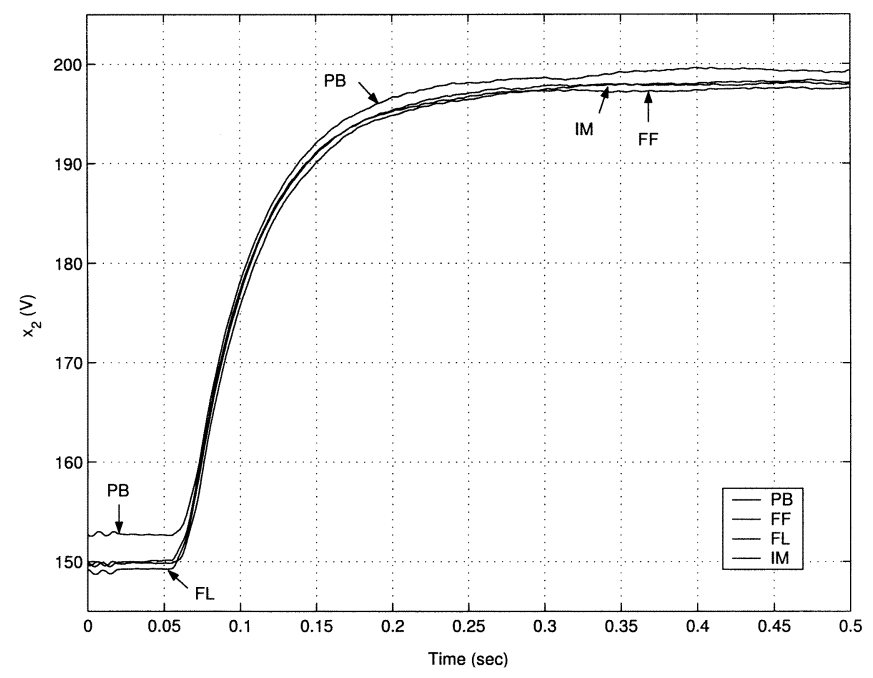

Fig. 3. Response of the output voltage to a reference change from 160 to 200 $\mathrm{V}$ applied at $t=0.05 \mathrm{~s}$ (the known parameter case).

output voltage reference change from 160 to 200 V, while Fig. 4 shows the harmonic content of the input current for each controller. We see that the transient behavior of the controllers is almost identical. The PB controller has slightly smaller steadystate error, but larger fourth-order harmonics, owing to the auxiliary dynamics. The steady-state error in all cases is significant, emphasizing the need for parameter adaptation.

Table II shows the harmonic characteristics for each case, namely the dc voltage error, the displacement angle, the total harmonic distortion (THD) and the power factor (see [1] for definitions). ${ }^{9}$ We see that the highest power factor and the lowest THD are achieved by the PB controller. The FL controller achieves the smallest displacement ${ }^{10}$ but with higher THD. On the other hand, the FL controller is the simplest one to implement, since it does not require the derivative of $x_{1}^{\star}$ and also it does not depend on the parameters $L$ and $C$ (note, however, that the other control schemes, which depend on $L$ and $C$ are not significantly sensitive to these parameters). Fig. 5

\footnotetext{
${ }^{9}$ The waveform of the first harmonic of the input current, which is required in order to compute these values, was estimated from the input current waveform, using the least-squares method.

${ }^{10}$ This is due to the fact that the negative phase shift introduced by FL (see (17)) compensates for the positive phase shift of the actual system, which may be caused by unmodeled dynamics and/or time delays.
} 


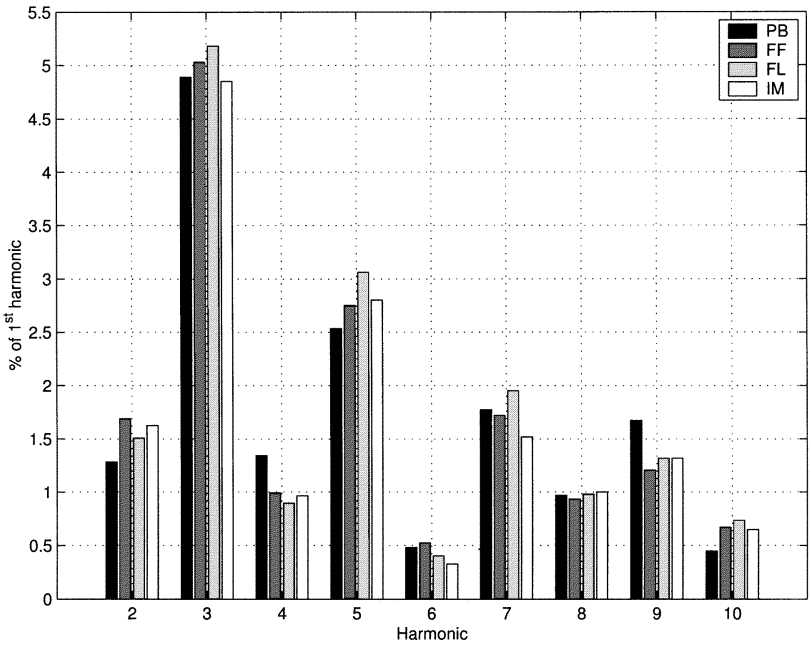

Fig. 4. Amplitude of the input current harmonics for $R=87 \Omega$.

TABLE II

HARMONIC ANALYSIS RESULTS FOR $R=87 \Omega$

\begin{tabular}{c|c|c|c|c}
\hline Control & $\begin{array}{c}\text { DC error } \\
(\mathrm{V})\end{array}$ & $\begin{array}{c}\text { Displace- } \\
\text { ment }\left({ }^{\circ}\right)\end{array}$ & $\begin{array}{c}\text { THD } \\
(\%)\end{array}$ & $\begin{array}{c}\text { Power } \\
\text { factor }\end{array}$ \\
\hline \hline PB & 0.75 & 1.47 & 7.53 & 0.9969 \\
\hline FF & 2.59 & 1.71 & 7.94 & 0.9964 \\
\hline FL & 1.95 & -0.49 & 8.88 & 0.9960 \\
\hline IM & 2.11 & 0.68 & 8.15 & 0.9966 \\
\hline
\end{tabular}
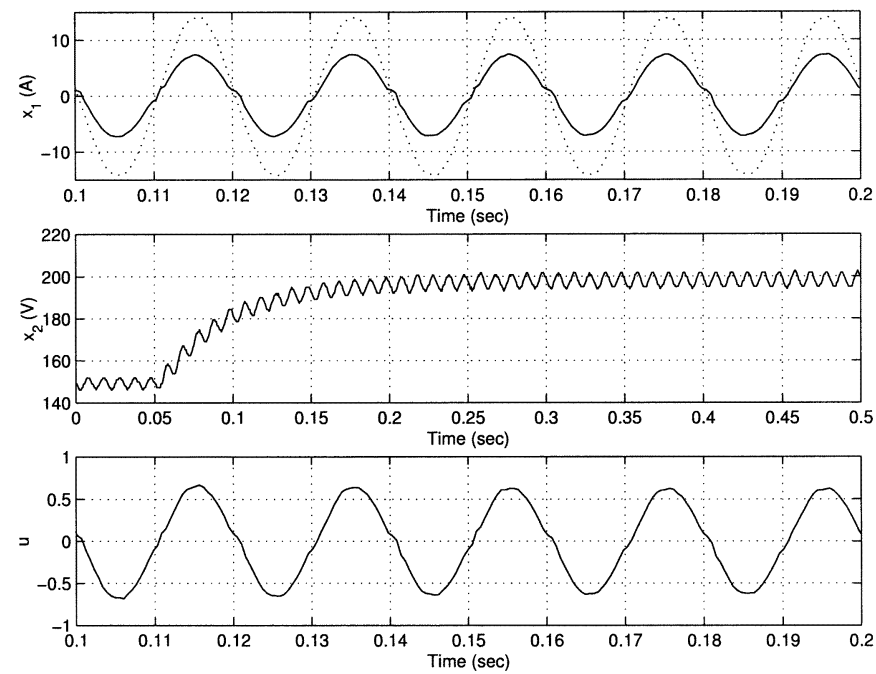

Fig. 5. Response to an output voltage reference change for the FL controller. Top graph: input current (solid line) and input voltage (dotted line). Middle graph: output voltage. Bottom graph: control input.

shows the input current, output voltage and control input for the FL case.

\section{B. The Unknown Parameter Case}

In this section, we test the controllers with the adaptation schemes proposed in Section III-B. In particular, for the PB controller we apply the PB estimator, while for the FF, FL, and IM controllers we employ alternatively the NLPI or the I\&I adaptation. The design parameters are given in Table III. For the sake
TABLE III

PARAMETER VALUES FOR THE PB, NLPI, AND I\&I ESTIMATORS

\begin{tabular}{c|l}
\hline Adaptation & Parameters \\
\hline \hline $\mathrm{PB}$ & $\gamma=1 \cdot 10^{-3}$ \\
\hline $\mathrm{NLPI}$ & $\alpha=5, \beta=0.05$ \\
\hline $\mathrm{II}_{1}$ & $\kappa=0, \lambda=2 \cdot 10^{-4}$ \\
\hline $\mathrm{II}_{2}$ & $\kappa=0.01, \lambda=2 \cdot 10^{-4}$ \\
\hline
\end{tabular}

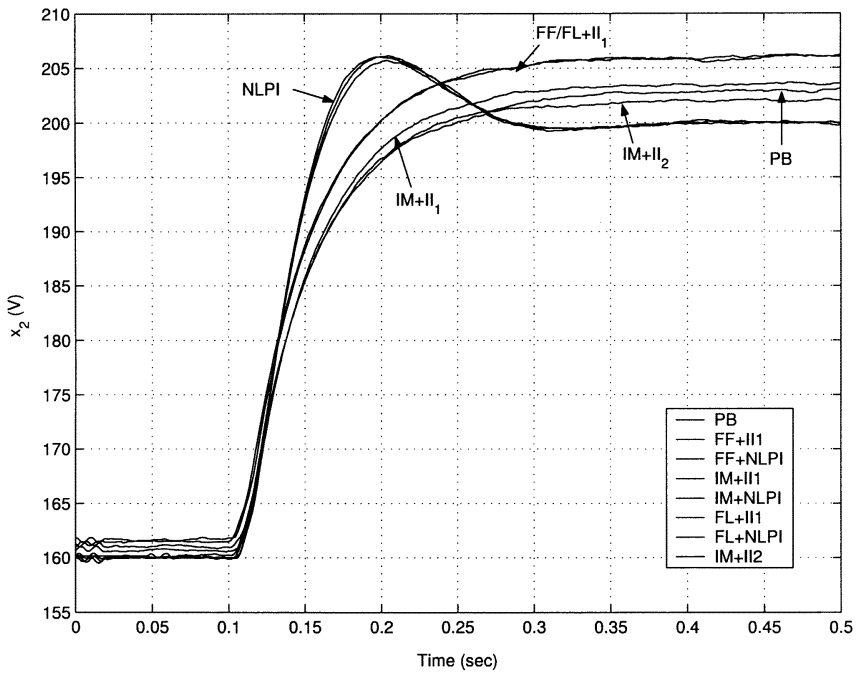

Fig. 6. Response of the output voltage to a reference change from 160 to 200 $\mathrm{V}$ applied at $t=0.1 \mathrm{~s}$ (the unknown parameter case).

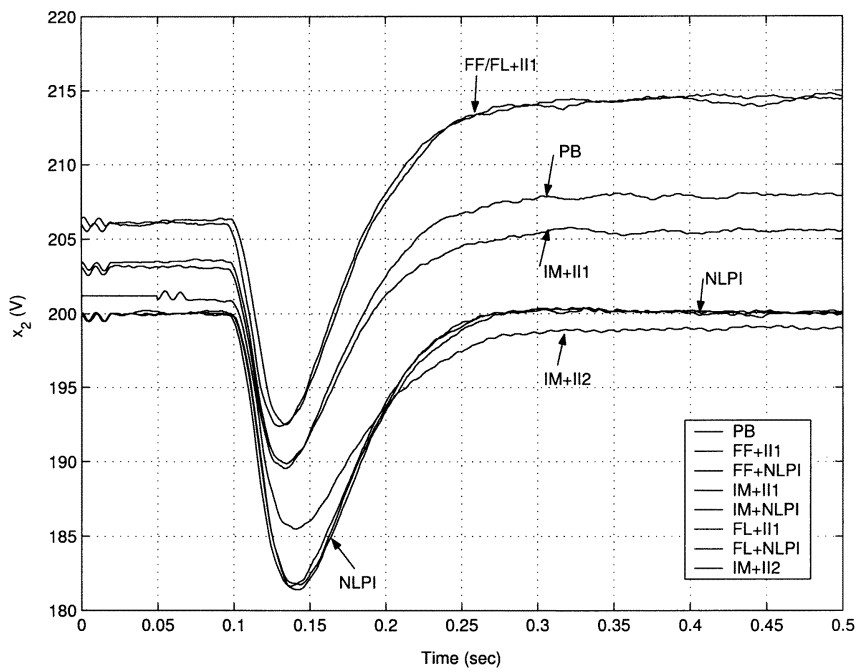

Fig. 7. Response of the output voltage to a load change from 87 to $51 \Omega$ applied at $t=0.1 \mathrm{~s}$ (the unknown parameter case).

of comparison, we consider mainly the case (denoted by $\mathrm{II}_{1}$ ) where the I\&I adaptation is applied only on $R$ (i.e., $\kappa=0$ ). The full $\mathrm{I} \& \mathrm{I}$ adaptation scheme (denoted by $\mathrm{II}_{2}$ ) is considered only with the IM controller.

Fig. 6 shows the filtered response to an output voltage reference change from 160 to $200 \mathrm{~V}$. We see that the NLPI control scheme achieves the smallest steady-state error (owing to the integral action), but with a large overshoot. On the other hand, the PB and I\&I estimators do not affect the response, which evolves as in the known parameter case. 


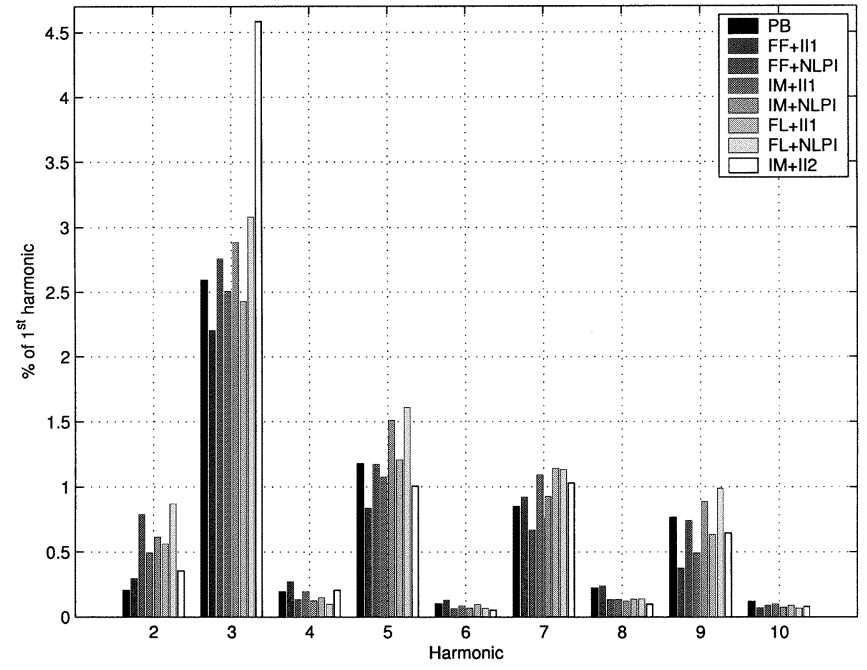

Fig. 8. Amplitude of the input current harmonics for $R=51 \Omega$.

TABLE IV

HARMONIC ANALYSIS RESULTS FOR $R=51 \Omega$

\begin{tabular}{c|c|c|c|c}
\hline Control & $\begin{array}{c}\text { DC error } \\
(\mathrm{V})\end{array}$ & $\begin{array}{c}\text { Displace- } \\
\text { ment }\left(^{\circ}\right)\end{array}$ & $\begin{array}{c}\text { THD } \\
(\%)\end{array}$ & $\begin{array}{c}\text { Power } \\
\text { factor }\end{array}$ \\
\hline \hline $\mathrm{PB}$ & 8.00 & 1.76 & 5.40 & 0.9981 \\
\hline $\mathrm{FF}+\mathrm{II}_{1}$ & 14.36 & 2.33 & 5.50 & 0.9977 \\
\hline $\mathrm{FF}+\mathrm{NLPI}$ & 0.04 & 2.81 & 5.74 & 0.9972 \\
\hline $\mathrm{IM}+\mathrm{II}_{1}$ & 5.56 & 1.92 & 5.73 & 0.9978 \\
\hline $\mathrm{IM}+\mathrm{NLPI}$ & 0.04 & 2.17 & 6.18 & 0.9974 \\
\hline $\mathrm{FL}+\mathrm{II}_{1}$ & 14.63 & 0.00 & 6.30 & 0.9980 \\
\hline $\mathrm{FL}+\mathrm{NLPI}$ & 0.04 & 0.55 & 6.12 & 0.9981 \\
\hline $\mathrm{IM}+\mathrm{II}_{2}$ & 0.95 & 1.65 & 7.11 & 0.9971 \\
\hline \multicolumn{4}{|r|}{}
\end{tabular}

Fig. 7 shows the filtered output voltage response to a load change from 87 to $51 \Omega$, while Fig. 8 shows the harmonic content of the input current for various control schemes. We see that the I\&I (with adaptation on $R$ only) and the PB estimators are slightly faster than the NLPI controller. However, there is a significant steady-state error, which changes when we perturb the load. This is mostly due to the uncertainty of the parameter $r$ and to unmodeled dynamics. Applying the I\&I adaptation on both $R$ and $r$ partially compensates for this uncertainty. However, the adaptation on $r$ increases the third-order harmonics.

Table IV shows the harmonic characteristics for each case. Again we see that the NLPI controller leads to the smallest steady-state error and, in combination with the FL control scheme, achieves the highest power factor. Fig. 9 shows the input current, output voltage and the estimated parameter $I_{d}$ for the FL + NLPI case.

Finally, Fig. 10 shows the harmonic content of the input current for the $\mathrm{IM}+\mathrm{II}_{2}$ case compared to the European standard EN61000-3-2, which specifies the harmonic limits for equipment drawing up to $16 \mathrm{~A}$ per phase. We conclude that all controllers proposed here achieve with a large margin the requirements imposed by this standard.

\section{CONCLUSION}

In this paper, an experimental comparison of several control schemes for a PFP circuit has been presented. The results have
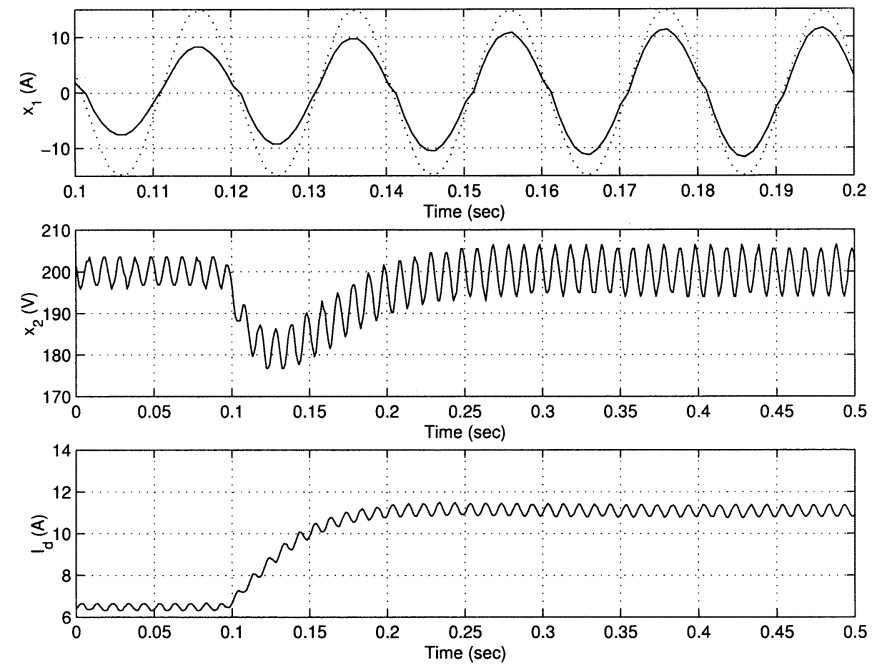

Fig. 9. Response to a load change for the FL + NLPI controller. Top graph: input current (solid line) and input voltage (dotted line). Middle graph: output voltage. Bottom graph: estimated parameter $I_{d}$.

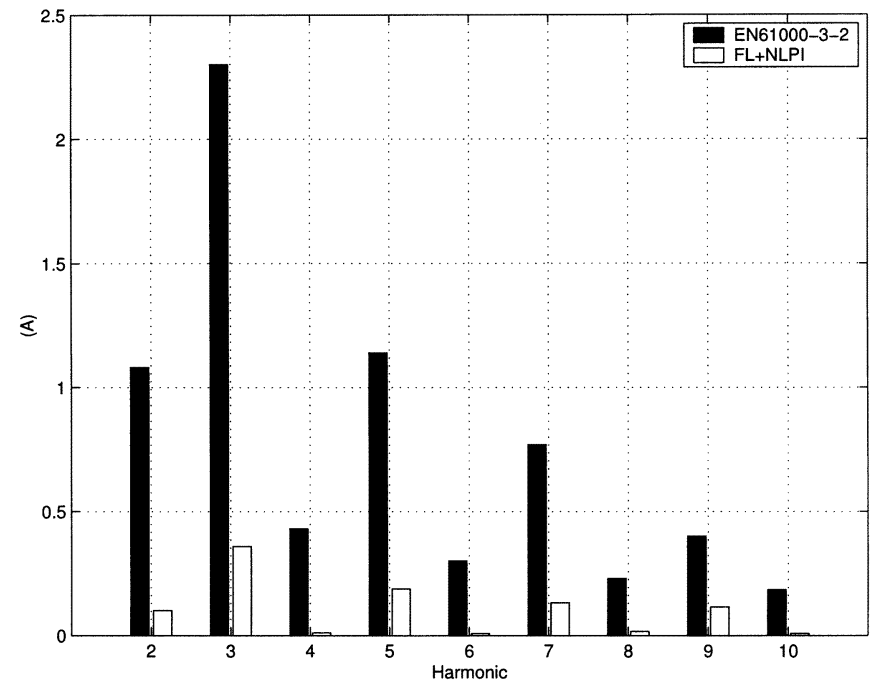

Fig. 10. Amplitude of the input current harmonics for the $\mathrm{IM}+\mathrm{II}_{2}$ controller compared to the standard EN61000-3-2 (the amplitude of the first harmonic is 12 A).

shown that when the load $R$ is known, the PB controller achieves the highest performance and robustness. If $R$ is unknown and an adaptation mechanism on $R$ is employed, the PB and IM control schemes are the most robust against changes in the load, at the expense of higher complexity. To further improve robustness, it is necessary to make use of a different adaptation scheme. Namely, it is necessary to adapt the parameter $I_{d}$. If such an adaptation is used, the FF, IM, and FL controllers are (in steady state) almost insensitive to load changes. However, the transient response is slightly degraded. Finally, the FL control scheme is the easiest to implement, since it uses only static feedback and does not require the derivative of $x_{1}^{\star}(t)$. The tradeoff is slightly higher harmonics and a small negative phase shift of the input current. ${ }^{11}$ Further study is in progress to extend the proposed methodologies to more complex converters.

${ }^{11}$ However, as noted in Section IV-A, this last property may actually improve the power factor. 


\section{REFERENCES}

[1] N. Mohan, T. A. Undeland, and W. P. Robbins, Power Electronics: Converters, Applications and Design. New York: Wiley, 1995.

[2] R. Morici, C. Rossi, and A. Tonielli, "Variable structure controller for AC/DC boost converter," in 20th Int. Conf. Ind. Electron., Contr. Instrument., 1994, pp. 1449-1454.

[3] J. Kassakian, M. Schlecht, and G. Verghese, Principles of Power Electronics. Reading, MA: Addison-Wesley, 1991.

[4] G. Escobar, D. Chevreau, R. Ortega, and E. Mendes, "An adaptive passivity-based controller for a unity power factor rectifier," IEEE Trans. Contr. Syst. Technol., vol. 9, pp. 637-644, 2001.

[5] R. Ortega and A. Astolfi, "Nonlinear PI control of uncertain systems: an alternative to parameter adaptation," in 40th IEEE Conf. Decision Contr., Orlando, FL, 2001, pp. 1749-1754.

[6] A. Astolfi and R. Ortega, "Immersion and invariance: a new tool for stabilization and adaptive control of nonlinear systems," in IFAC Symp. Nonlinear Contr. Syst. Design, St. Petersburg, Russia, 2001, pp. 81-86.

[7] H. Khalil, Nonlinear Systems, 3rd ed. Englewood Cliffs, NJ: PrenticeHall, 2002. 